\title{
Veterinary Osseous Site Reconstruction Utilizing Autologous Dentin from Extracted Teeth
}

\author{
${ }^{1}$ Rocco E Mele, ${ }^{2}$ Gregori M Kurtzman, ${ }^{3}$ Itzhak Binderman, ${ }^{4}$ Lanka Mahesh
}

\begin{abstract}
Following tooth extraction, we often observe significant changes of ridge contour that is the result of alveolar bone loss. Most of the bone loss occurs during the first 3 to 4 months following extraction. To prevent this, it is strongly recommended to graft the extraction site with a biocompatible and bioactive osseous graft material at the time of extraction. There are many options available for socket grafting each with its own deficiencies. Most of the synthetic and allograft type bone substitutes preserve the alveolar ridge during the repair phase of the wound healing, but subsequently resorb during the following remodeling phase and therefore only achieve partial ridge restoration. Xenografts, on the contrary, do not osseointegrate sufficiently and form "islands" of foreign body within the bone structure. Recently, a novel procedure was developed where the extracted tooth is immediately processed into an autologous graft that preserves the alveolar ridge for many years and is biocompatible to the host site in a highly predictable manner. The procedure also helps to keep treatment costs lower than prepackaged graft materials. This autologous dentin particulate, made from the extracted tooth of the patient, undergoes ankylosis with the newly formed bone around it. The result is a biological connection or fusion of the graft and the host bone, an interface that is more biological than other alternatives, hence providing optimal and predictable results in the short and long term.
\end{abstract}

Keywords: Autologous grafting, Autologous particulate dentin graft, Dentin graft, Smart dentin grinder, Socket preservation, Tooth-derived bone graft.

How to cite this article: Mele RE, Kurtzman GM, Binderman I, Mahesh L. Veterinary Osseous Site Reconstruction Utilizing Autologous Dentin from Extracted Teeth. Int J Oral Implantol Clin Res 2017;8(2\&3):72-80.

Source of support: Nil

Conflict of interest: None

\section{INTRODUCTION}

The healing process of a tooth extraction site results in significant changes to the ridge contour that is the result

\footnotetext{
1,2,4 Private Practitioner, ${ }^{3}$ Professor

${ }^{1}$ Veterinary Medicine, Tuson, Arizona, USA

${ }^{2}$ Silver Spring, Maryland, USA

${ }^{3}$ Department of Dentistry, Tel Aviv University, Tel Aviv, Israel

${ }^{4}$ Department of Implantology, The Specialist Clinic, New Delhi India

Corresponding Author: Gregori M Kurtzman, Private Practitioner, Silver Spring, Maryland, USA, Phone: +3015983500 , e-mail: drimplants@aol.com
}

of alveolar bone loss typically at a larger extent on the labial/buccal than on the lingual/palatal. This is mainly due to the relative osseous density differences. ${ }^{1}$ The majority of this accelerated bone loss occurs during the first 3 months following tooth extraction and gradually slows down over the ensuing months. Bone resorption of such nature may lead to a reduction of as much as 50\% in the width of the alveolar ridge within 12 months. ${ }^{2}$ Moreover, hard tissue loss due to remodeling continues for many years although at a slower pace. The labial bone wall thickness has been identified as the most critical factor influencing bone resorption. This becomes more critical in the frontal and premolar area of the jaw where typically a thin buccal lamella is present. It is reasonable to assume that resorption of buccal plates of the alveolar ridge is greater since most of this bone is formed during tooth eruption and has a lower density then bone located on the lingual aspect.

Significant changes of the ridge contour, including the soft (mucosal) tissues, are a major cause of esthetic and functional deficiencies. In addition, using dental implants for prosthetic reconstruction will often not be possible due to such deficient alveolar ridge anatomy. There is sufficient compelling evidence that immediate grafting of extraction sockets by various ridge preservation techniques, including the placement of graft materials and/or the use of occlusive membranes, limits or even prevents alveolar bone loss during the repair phase of wound healing. ${ }^{3}$ Socket restoration techniques for ridge preservation utilizing different biomaterials and/or barrier membranes often result in better maintenance of tissue volume. Still, the inevitable biological process of postextraction bone resorption and bone remodeling cannot be stopped due to lack of internal loading within the bone that is transmitted by occlusal loading on teeth or implants that aid in bone maintenance. ${ }^{4}$ The best results in minimizing resorption in such cases have been demonstrated by using autologous dentin grafts that interact differently than nonautologous grafts. ${ }^{5,6}$

Tooth dentin and cementum, being similar in their mineral and protein composition to membranous bone, are inductive to osteogenic cells by directly depositing new bone matrix on their surfaces, resulting in ankylosed dentin-bone interfaces. Such ankylosed interface occurs when avulsed teeth are reimplanted back into their 
sockets $^{7-9}$ or when extracted teeth are processed immediately after being extracted into a particulate dentin graft that is inserted into the freshly extracted socket in the same patient. ${ }^{5,6}$

The alveolar bone, during its initial healing phase, reacts more favorably to an ankylosis process than it would to osseointegration only. Recent clinical results show optimal alveolar ridge contour preservation over 5 years postsurgical follow-up when decoronation of ankylosed teeth is performed. ${ }^{10,11}$ In a recent study, a series of cases reported a technique in which the extraction socket was restored with autologous, particulated mineralized dentin that was placed immediately after tooth extraction. ${ }^{5,12,13}$ In all cases, patients exhibited a stable volume of soft and hard tissues and good osseointegration of titanium implants that were placed in this augmented socket. ${ }^{5,12}$ Herein, we describe the efficacy and safety of this technique using the smart dentin grinder (SDG) (KometaBio, Cresskill, NJ) procedure for ridge preservation after extraction of teeth.

Adopting an effective protocol for preservation of alveolar ridge is not only important for implant-supported prosthesis, but also imperative in order to restore surgically produced defects that may result from removal of impacted teeth or support dehiscent root surfaces of periodontally involved teeth. Since every tooth extraction induces loss of alveolar bone, it is indicated to process the extracted tooth into a particulate graft whenever possible in order to preserve the alveolar bone.

\section{THE CLINICAL PROCEDURE}

\section{Procedure Safety}

The SDG procedure (Fig. 1) was developed by the KometaBio company and is indicated for the same patient's teeth (dog/cat) to be used at the time of extraction. The extracted tooth is usually covered with a biofilm

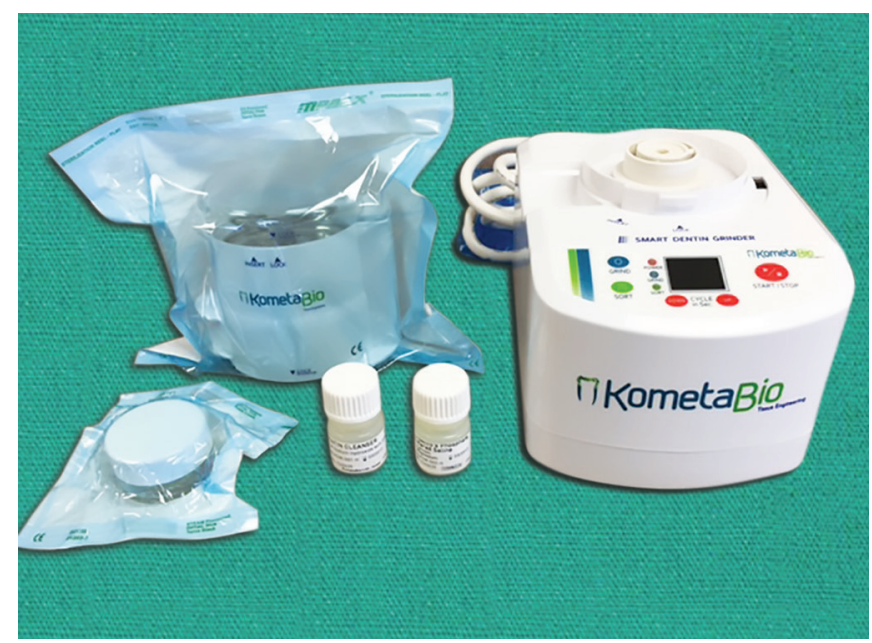

Fig. 1: The SDG unit (KometaBio) of bacteria, toxins, and soft tissue, such as periodontal ligament on its root surfaces. Following extraction, all foreign materials should be thoroughly removed using a diamond bur in a handpiece. "Shaving" of the organic coat of the root surfaces which includes the periodontal ligament and associated bacterial biofilm is easily removed by a carbide bur (Fig. 2).

At the end of this phase (2 minutes), a very clean surface of tooth structure that consists of dentin is achieved. A thin layer of cementum is usually removed and most of the enamel is stripped from the extracted tooth along with any carious tooth structure (Fig. 3). Before placing teeth into the sterile grinder chamber, they should be thoroughly dried with the air syringe. The grinding and sorting process into specific particulate size takes approximately 1 minute. The grinding of the tooth/teeth takes 3 seconds and vibration for sorting of the ground particles takes 20 seconds. More than $90 \%$ of the particulate accumulates in the special "chamber drawer" that contains particulate of the size of 300 to $1200 \mu \mathrm{m}$, the optimal size for achieving an osteogenic interaction at the grafted site. It is noted that the particulate volume will be about 2 to 3 times more than

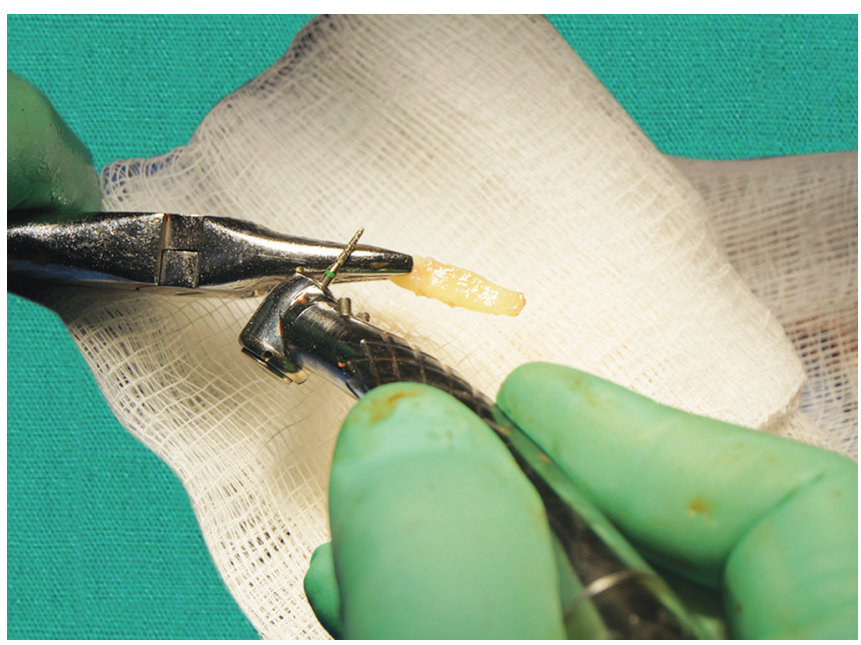

Fig. 2: Stripping the cementum and periodontal ligament from the extracted tooth with a dental handpiece and bur

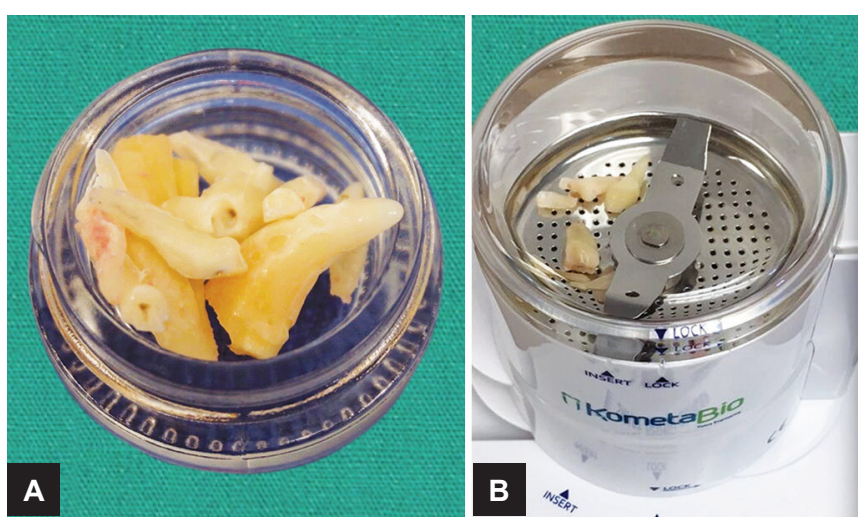

Figs $3 A$ and $B:(A)$ Prepared extracted teeth following stripping of cementum from the roots; (B) Inserted into the SDG ready to start processing 


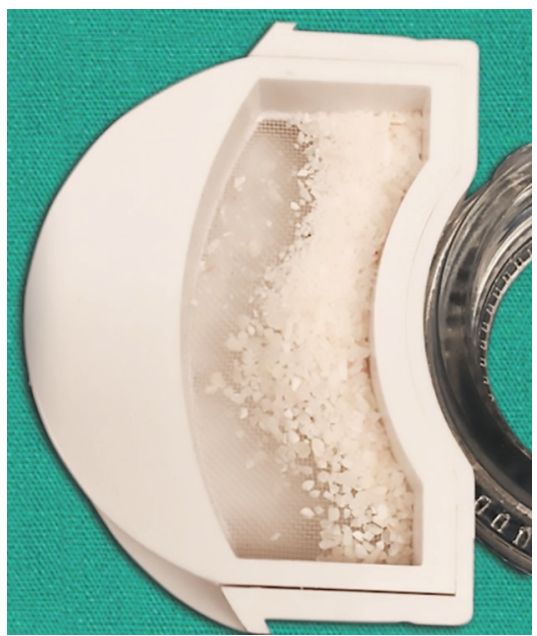

Fig. 4: Extracted teeth processed in the SDG

the volume of the tooth prior to grinding (Fig. 4). Next, the particulate is poured into a sterile small glass container and a chemical cleanser consisting of $0.5 \mathrm{M} \mathrm{NaOH}$ and $20 \%$ ethanol is poured in to cover the particulate. Some gentle shaking helps the cleanser solution to wet the particles and penetrate into the dentin tubules. It is important to note that the cleanser is very effective in dissolving all organic materials, like bacteria, endotoxins, viruses on the particle surface as well as within the dentinal tubules.

Following immersion in the cleanser for 10 minutes, the cleanser is decanted by absorbing the liquid with sterile gauze. A phosphate buffer saline (PBS) solution is poured into the dish and agitated for 3 minutes with gentle shaking. The PBS washes out the remaining cleanser, adjusting the $\mathrm{pH}$ back to physiological $\mathrm{pH}$ of 7.2. Those treatment times are optimized to achieve complete removal of all organic substances from the particulate surfaces. Next, the PBS is absorbed into sterile gauze and the slightly wet particulate is ready to be grafted (Fig. 5). At this stage, it is possible that a few bacteria may survive the cleansing procedure; however, these bacteria are the patient's own bacteria. The patient's normal oral bacterial environment is normally protective for the patient. The dentist should be aware that the bacterial biofilm that is concentrated in the gingival crevice can produce growth of an anaerobic bacterial environment.

\section{Procedure Effectiveness}

Dentin, cementum, and membranous bones (mandible and maxilla) develop from unique embryonic neural crest cell populations. Therefore, dentin and membranous bone consist of similar molecular cues and structures. These tissues consist of hydroxyapatite (HA) mineral that is deposited on and between type I collagen molecules, inside fibers as well as extrafibrillar HA, surrounding

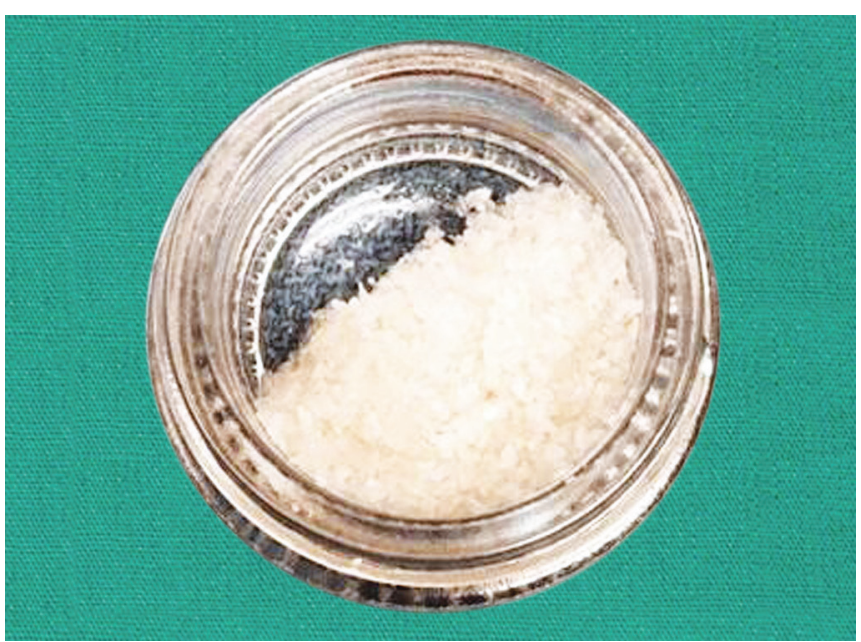

Fig. 5: Processed extracted teeth following rehydration demonstrating an increase in volume compared with the dry processed teeth

these collagen fibers. In addition, the noncollagen fraction of glycoproteins, although being only a small fraction of the matrix volume, are vastly important in regulating mineralization, attraction of osteoprogenitor cells and inductive molecules like bone morphogenetic protein.

This makes the matrix very bioactive and most importantly initiating regeneration of bone or repair of cementum and dentin. It is evident that teeth that are extracted by injury, also called avulsed teeth, when reimplanted into their sockets or transplanted teeth, undergo either partial or total ankyloses and may function for many years. Root ankylosis is a biological repair process where the root cementum and dentin surfaces are an attractant to osteogenic cells that attach to root surface through an integrin-arganine-glycerineaspartate covalent biological attachment, wherein the dentin and cementum matrices are instructing local cells to differentiate into active osteoblast that secrete mineralized matrix directly on dentin and cementum surfaces. This creates an ankylosis interface which cements the newly formed bone to the dentin surface. At the remodeling phase, the osteoclasts are unable to distinguish between bone and dentin matrices as both have a similar embryological origin. ${ }^{14}$

Extracted teeth that are processed as described above are inductive to the osteogenic environment in the fresh socket and therefore new bone is deposited directly on the dentin particulate surfaces, leading to an ankylosed interface between the new bone and dentin particles. This process creates hard tissue (bone-dentin) connectivity that restores the alveolar ridge and preserves it for many years.

\section{Clinical Practicality}

The resulting graft material has been shown to produce highly predictable results in animal and human patients 
over the long term. The procedure of creating the graft material is not time consuming and only requires approximately 15 minutes to prepare and process the extracted teeth. Additionally, treatment costs are minimized vs using packaged osseous graft materials. Typically, healing time is also reduced due to the autologous nature of the graft which allows the clinician to complete the restorative and prosthetic procedure faster.

\section{CLINICAL CASES}

Extraction of teeth is an everyday practice of veterinary clinicians with most teeth extracted because of advanced bone loss due to prior periodontal disease. In most cases, root volume is sufficient to convert into particulate material as dentin recovered from the roots by the SDG procedure will result in 2 to 3 times the volume of the roots utilized.

\section{Case 1}

A 2-year-old F/S Welsh Corgi presented with need for an extraction of a compromised fractured \#208 and immediate grafting with autologous dentin prepared from that root.

\section{Results}

Bone-dentin tissue formed from autologous graft of dentin root particulate demonstrated a high stability of the site and ridge at 12 weeks (Figs 6 and 7).

\section{Case 2}

A 12-year-old F/S GS mix requiring multiple surgical extractions of \#s 404; 208 with site debridement and dentine autologous graft to augment and support the large maxillary and mandibular defects.

\section{Results}

Grafting of autogenous bone regenerated the normal bony anatomy with dentin autologous particulate supporting exceptional tissue maintenance (Figs 8 to 12).

\section{Case 3}

A 12-year-old F/S Husky with advanced periodontal disease and osteomyelitis (confirmed by histopathology). Treatment included surgical extraction of the affected teeth and removal of infected and necrotic tissue and bone as well as appropriate antibiotic treatment. Conventional
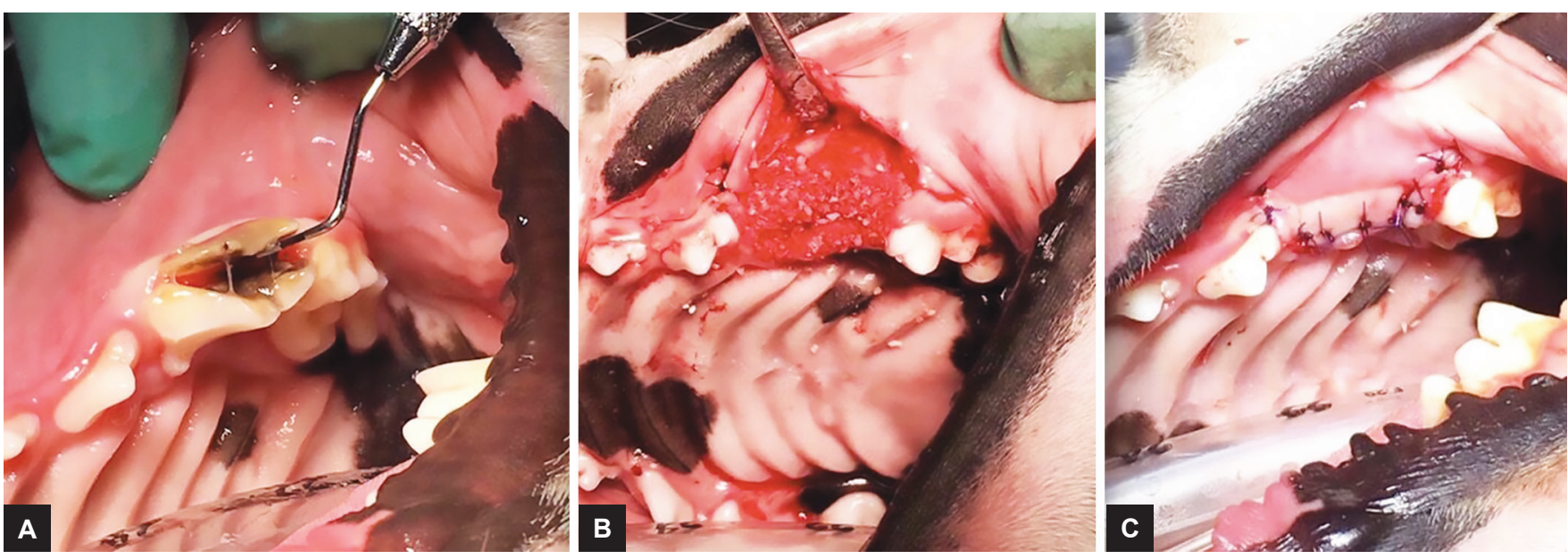

Figs 6A to C: (A) Buccal plate subgingival fracture; (B) Dentin particulate graft placed in prepared site;

(C) Site closed with sutures to get primary closure
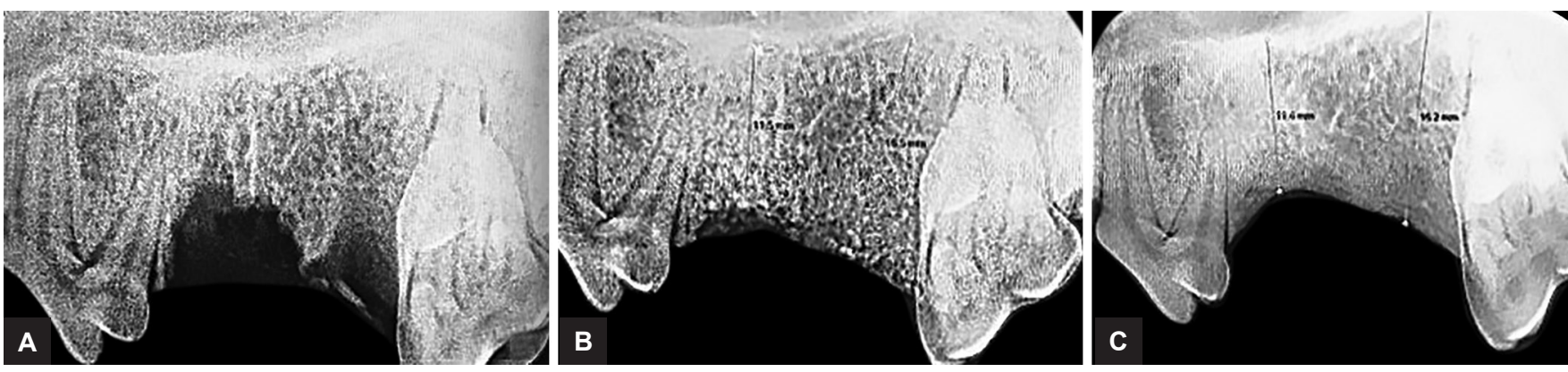

Figs 7A to C: (A) Extraction site; (B) Dentin graft placed, (C) 3-month postoperative follow-up 


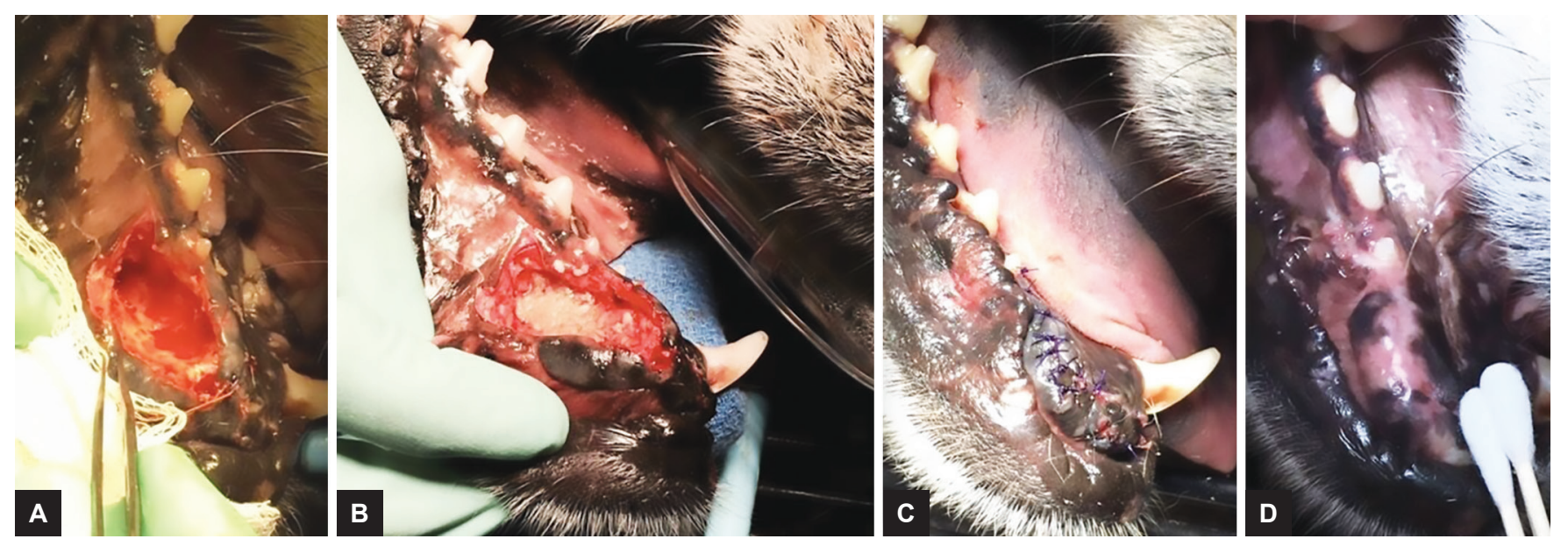

Figs 8A to D: (A) Surgical site development \#404; (B) Dentin autologous graft placed, (C) Primary closure of the site;

(D) Tissue maturation at 3 months postsurgery
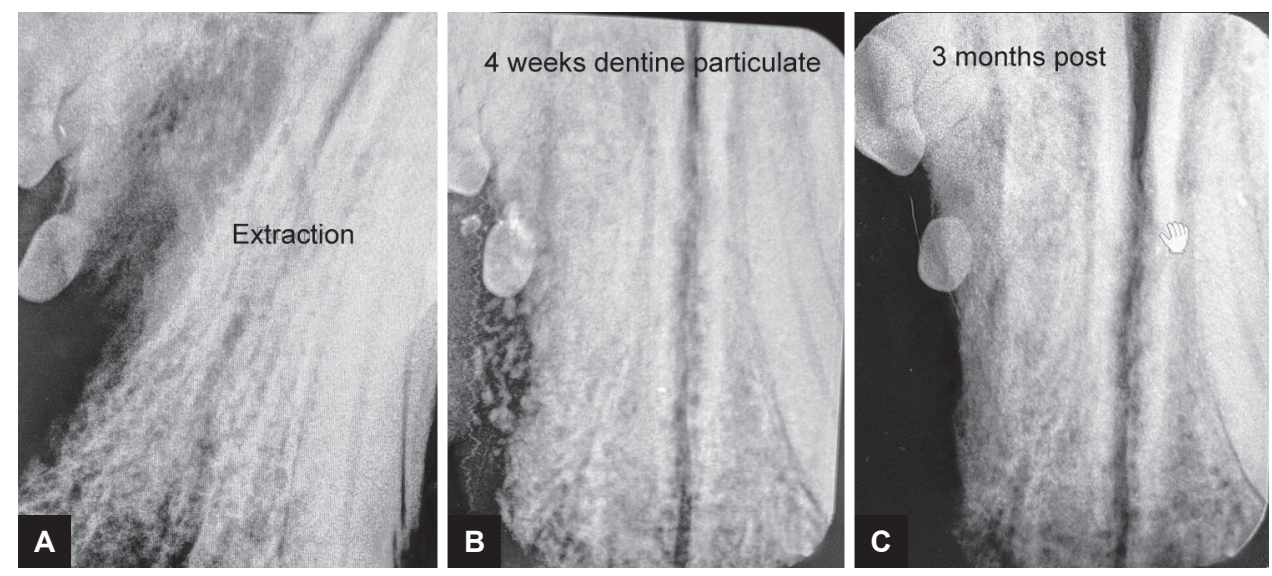

Figs 9A to C: (A) Radiograph of \#404 postextraction site; (B) 4 weeks postdentin grafting (C) 3-month follow-up demonstrating solid bone-dentin remodeling
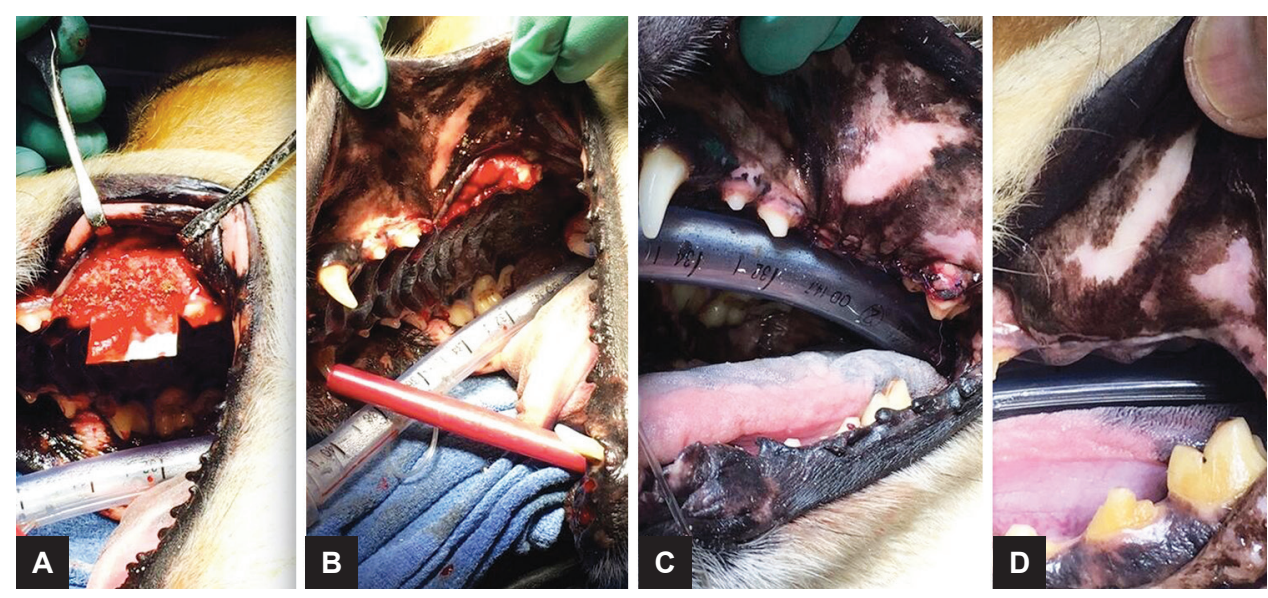

Figs 10A to D: (A) Extraction site \# 208; (B) Dentin grafting, primary closure; (C) Follow-up for suture removal; (D)12-week follow-up demonstrating healing with tissue maintenance

surgical debridement not only removes poorly vascularized (infected) bone but also brings well-vascularized tissue to the affected bone, facilitating the healing process and allowing antibiotics to reach the target areas. Dentin particulate was placed, filling the entire defect and a BioXclude membrane (Snoasis Medical, Golden, $\mathrm{CO}$ ) was utilized to provide graft stability and space maintenance needed to obtain ideal bone production.

\section{Results}

Serial radiographic study and clinical presentation displaying predictable and exceptional bone-dentin 

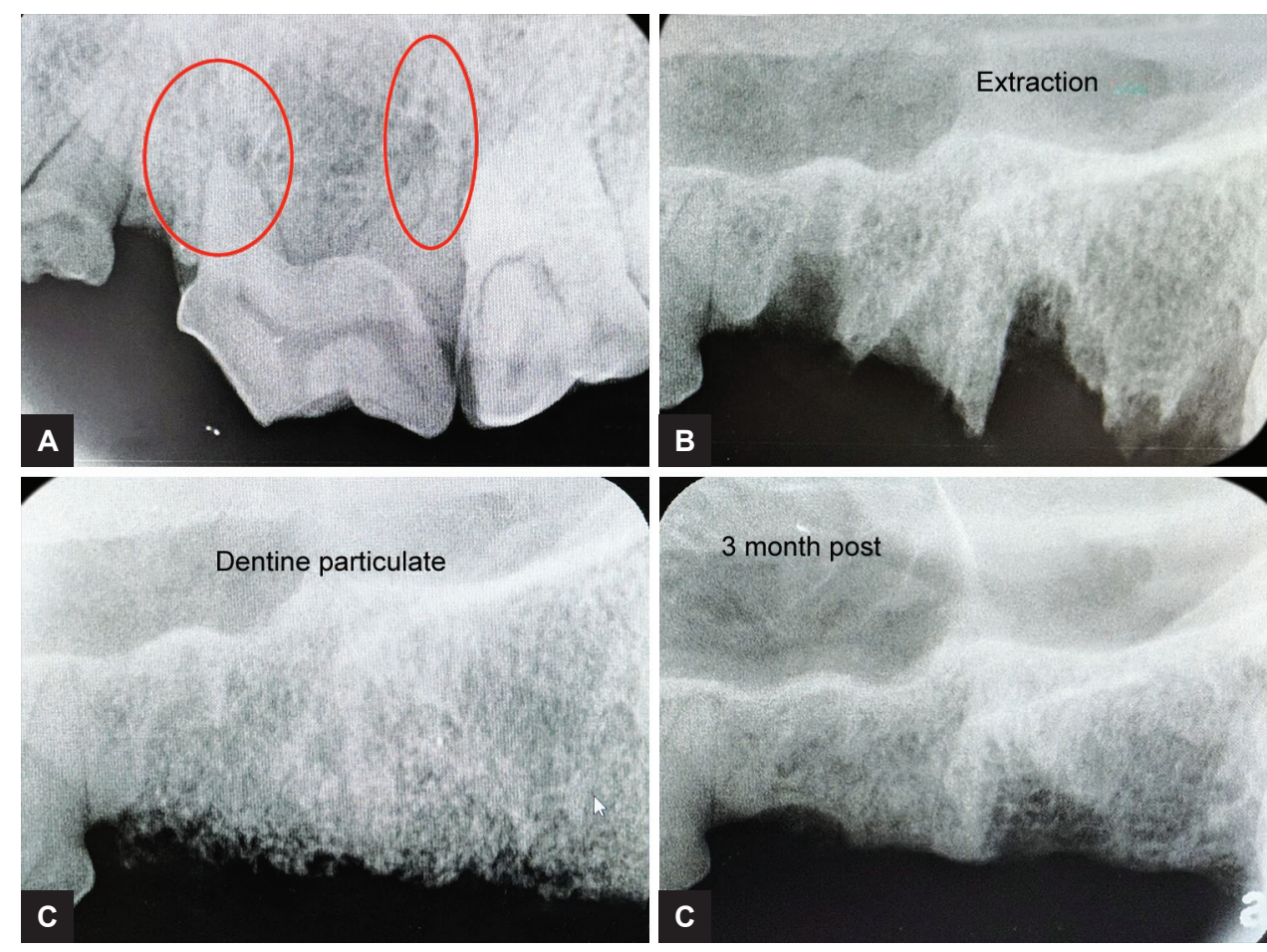

Figs 11A to D: (A) Sequential radiographs of \#208 preextraction; (B) Postextraction,

(C) Dentin graft placement; (D) 3-month follow-up
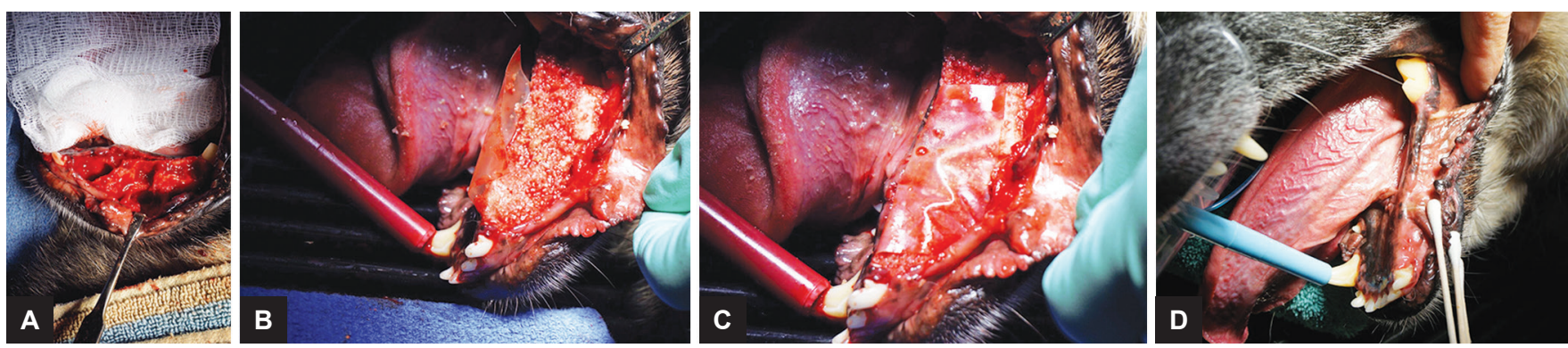

Figs 12A to D: (A) Hemi-mandible surgical site with affected teeth extracted and necrotic bone and tissue debridement,

(B) Dentin graft filling the defect; (C) Protective membrane placed, (D) Site closed with sutures

complex maturation and healthy oral mucosa (Figs 13 and 14).

\section{Case 4}

An 11-year-old M/N Akita with a mandibular distoocclusion (MAL 2) with severe palatal trauma resulting in communication between the oral cavity and the nasal sinus. Also present was palatal bone necrosis causing substantial palatal bone loss.

\section{Results}

Treatment included multiple surgical extractions with debridement and excision of the necrotic palatal bone and tissue. Extracted teeth were cleaned and ground into autologous particulate graft. A canine cortical strut (Calvaria) was prepared and stabilized with $2.0 \mathrm{~mm}$ cortical self-tapping screws to rebuild the lost palatal bone. Ossiflex bone membrane (Veterinary Transplant Services, Kent, WA) was utilized to close the oral nasal connection. Dentin graft filled the entire maxillary defect and a fascia lata membrane covered the reconstructed site before primary closure was completed (Figs 15 to 19).

\section{DISCUSSION}

The use of scaffolds for preserving or reconstructing the alveolar ridge or deficient site has become a frequent surgical procedure. Bone grafts usually undergo extensive remodeling and resorption during the first year after surgery. Unfortunately, this does not allow woven bone sufficient time to mature into lamellar bone and therefore immature bone will quickly resorb following scaffold resorption. This may affect the feasibility of an effective 


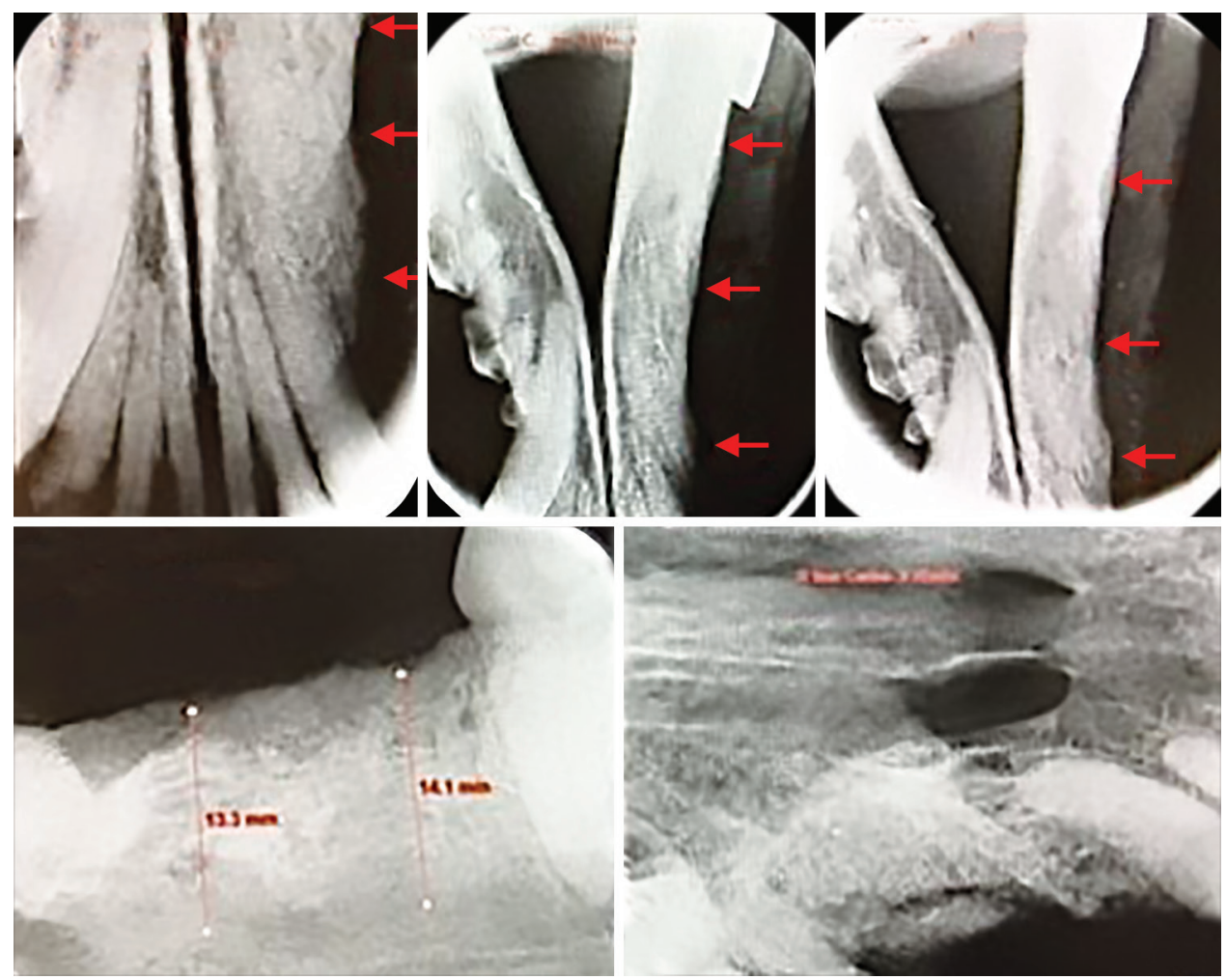

Fig. 13: Mandible restored to normal form and function at 9 weeks demonstrating bone remodeling (top 3), mandibular buccal view with increased vertical height development and ridge maintenance (bottom left) and maxillary canine (\#104) with bone fill at 9 weeks following dentin graft (bottom right)
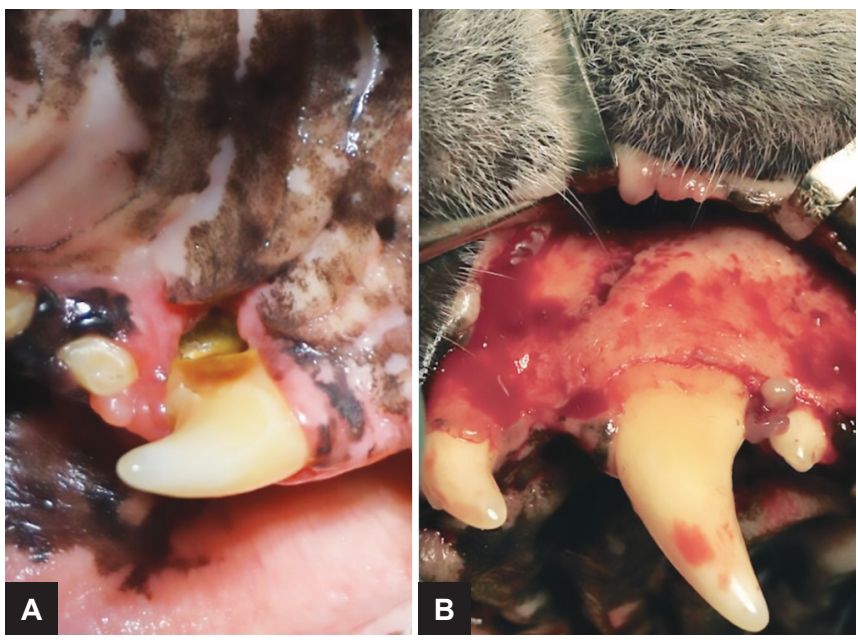

Figs 14A and B: (A) Palatal defect demonstrating necrosis of the hard palate; (B) buccal fenestration

rehabilitation. Some authors have suggested that graft resorption rate may be dependent on their embryologic origin, since grafts from membranous bone (i.e., calvarial or mandibular grafts) do not resorb as extensively as those from endochondral bone (as iliac crest bone grafts). Bone graft density may also be associated with their resorption, as it has been shown that high-density grafts undergo a slower and lower resorption than low-density grafts. It seems therefore that autogenic dentin-like membranous bone are optimal for long-standing preservation
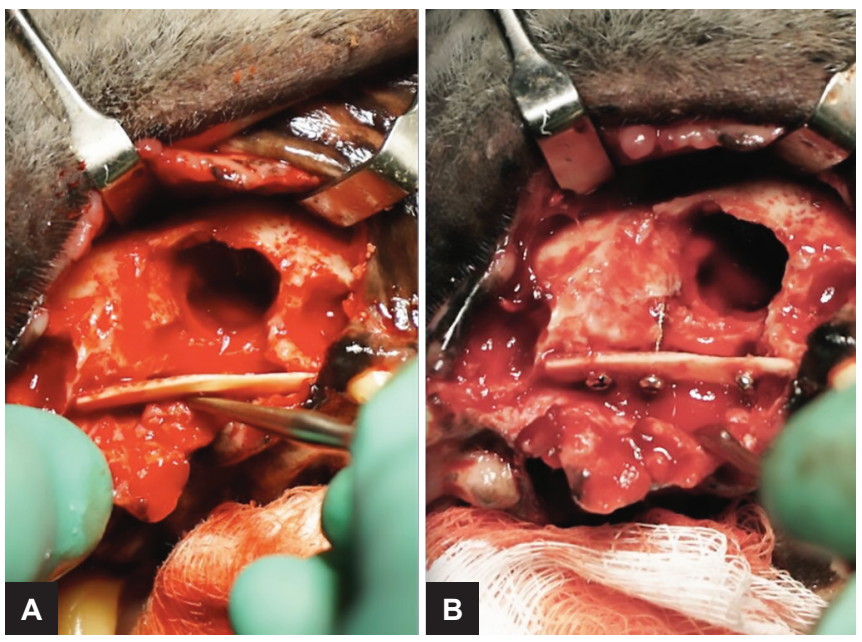

Figs 15A and B: (A) Cortical-cancellous strut stabilized with three cortical self-tapping screws; (B) The strut will replace the lost necrotic hard palate and support the dentin particulate graft

of augmented alveolar bone ridge. Autologous dentin and cementum undergo ankylosis when reimplanted back into osteogenic socket. Since they resorb slowly, and will take a few years to fully resorb, they maintain a strong scaffold and allow for sufficient time to achieve bone maturity.

Clinical studies of follow-up cases of reimplanted avulsed teeth or autotransplanted teeth from one site into surgically prepared sockets showed an immediate cellular interaction with dentin or cementum root 


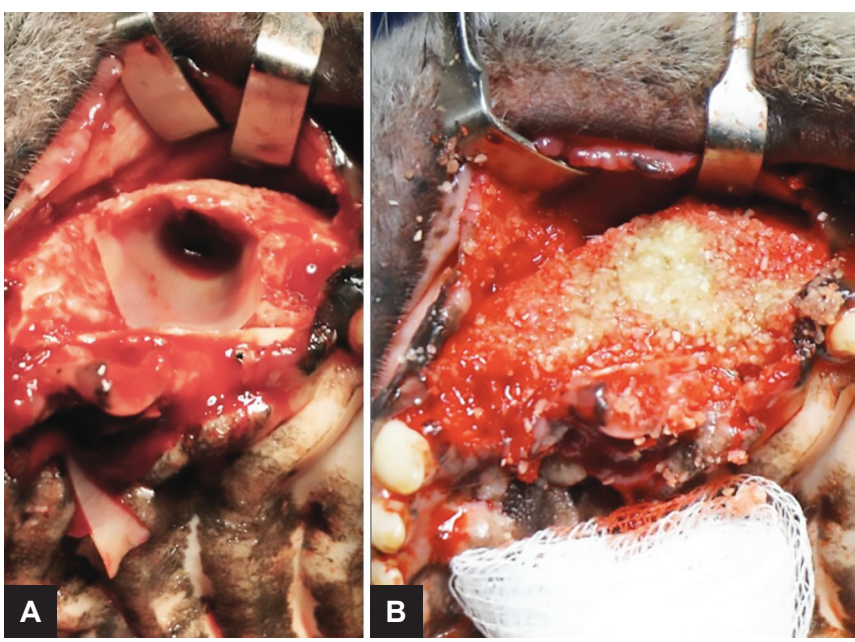

Figs 16A and B: (A) Ossiflex bone membrane "snow cone" shaped and placed into the oronasal communication to plug the defect; (B) Dentin graft placed to fill the entire defect

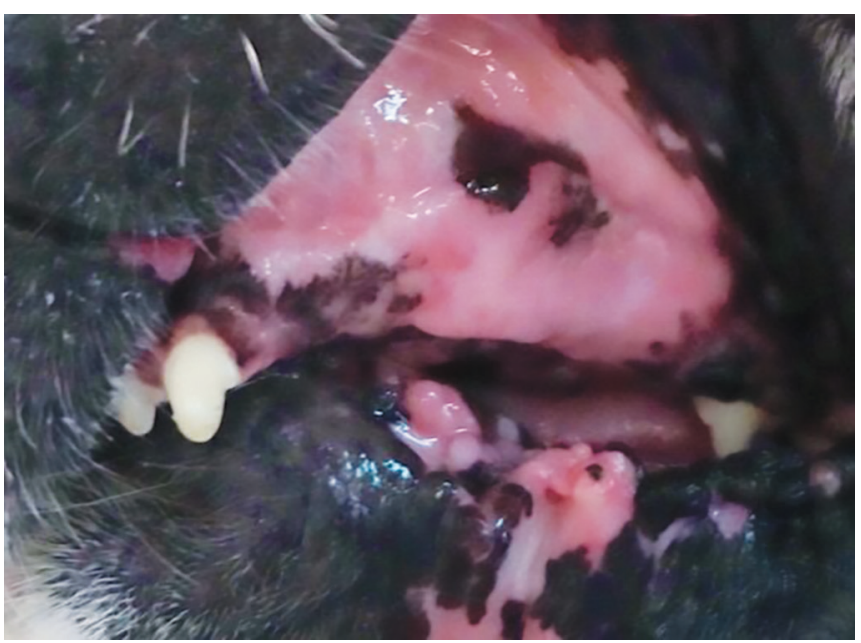

Fig. 18: Twelve-week post-op of the healed site

surfaces that results by cellular biologic attachment to their surfaces, differentiation into osteoblasts, and deposition of mineralized matrix directly on to dentin or cementum. This biological attachment is termed an ankylosis. It is important to note that such biologic interface between dentin or cementum mineralized matrices and the newly deposited mineralized bone matrix creates a functional biological connectivity that allows remodeling of all mineralized tissue. However, root resorption takes place in a very slow pace, and it is replaced by newly formed bone, thus preserving the alveolar ridge.

Malmgren ${ }^{10}$ has suggested to remove the coronal portion of ankylosed teeth in order to preserve the alveolar ridge. This concept of biological ankylosis of teeth is one and the same as what happens to autologous tooth particulate when the tooth undergoes an SDG process and then reimplanted back into the extraction site and/ or used for grafting alveolar bone defects and deficiencies. A recent study from Zurich University ${ }^{12}$ that used

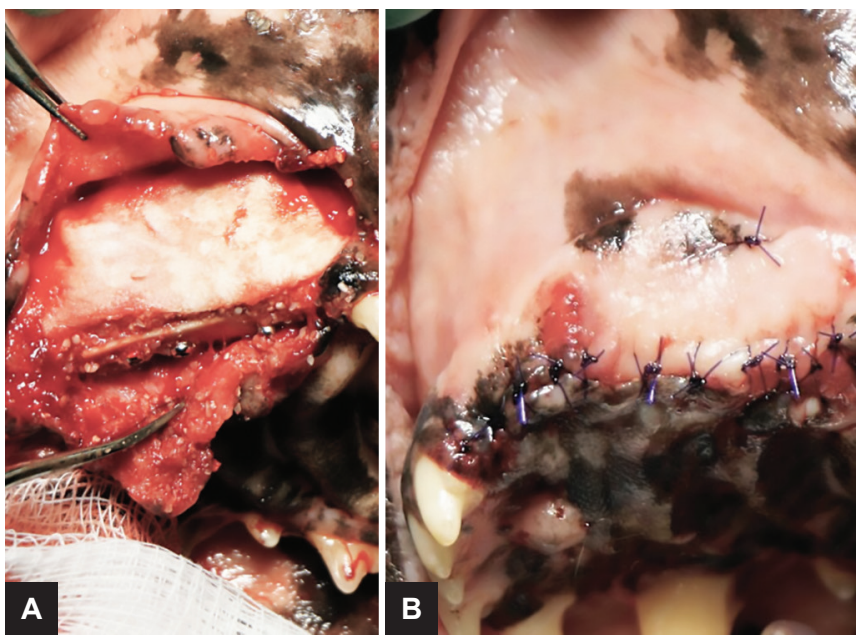

Figs 17A and B: (A) Fascia lata membrane placed to support the soft tissue and protect the grafted site; (B) Final closure with mattress and interrupted suture patterns to establish a tensionfree flap

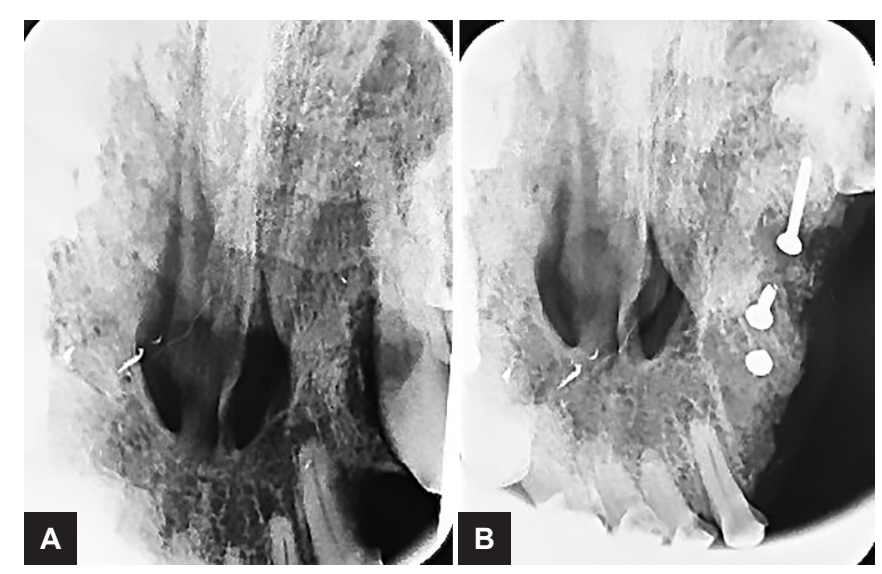

Figs 19A and B: (A) Radiographs demonstrating presurgical presentation; (b) 12 weeks posthealing with bone-dentin grafting, showing ridge maintenance

the same concept of grafting of particulate mineralized dentin found it safe and effective in preserving the alveolar ridge.

\section{CONCLUSION}

The extracted tooth in most cases being the patient's tissue can be transformed immediately into an osseous graft that will preserve the alveolar ridge and restore oral bony deficiencies' form and function. This protocol may be utilized instead of synthetic, allograft, or xenograft osseous graft materials with better predictable long-term results and lower treatment costs associated with use of these packaged graft materials.

\section{REFERENCES}

1. Pietrokovski J, Massler M. Alveolar ridge resorption following tooth extraction. J Prosthet Dent 1967 Jan;17(1):21-27.

2. Schropp L, WenzelA, Kostopoulos L, Karring T. Bonehealing and soft tissue contour changes following single-tooth extraction: 
a clinical and radiographic 12-month prospective study. Int J Periodontics Restorative Dent 2003 Aug;23(4):313-323.

3. Horowitz R, Holtzclaw D, Rosen PS. A review on alveolar ridge preservation following tooth extraction. J Evid Base Dent Pract 2012 Sep;12(3 Suppl):149-160.

4. Chappuis V, Araújo MG, Buser D. Clinical relevance of dimensional bone and soft tissue alterations post-extraction in esthetic sites. Periodontol 20002017 Feb;73(1):73-83.

5. Binderman I, Hallel G, Nardy C, Yaffe A, Sapoznikov L. Anovel procedure to process extracted teeth for immediate grafting of autogenous dentin. J Interdiscipl Med Dent Sci 2014 Oct;2:154.

6. Kosinski T. Innovative socket grafting techniques in preparation for dental implants. TPD 2017 Summer:22-27.

7. Andersson L, Blomlof L, Lindskog S, Feiglin B, Hammarstrom L. Tooth ankylosis. Clinical, radiographic and histological assessments. Int J Oral Surg 1984 Oct;13(5):423-431.

8. Andersson L. Dentin xenografts to experimental bone defects in rabbit tibia are ankylosed and undergo osseous replacement. Dent Traumatol 2010 Oct;26(5):398-402.
9. Andreasen JQ, Ravn JJ. Epidemiology of traumatic dental injuries to primary and permanent teeth in a Danish population sample. Int J Oral Surg 1972;1(5)235-239.

10. Malmgren B. Ridge preservation/decoronation. J Endod 2013 Mar;39(3 Suppl):S67-S72.

11. Filippi A, Pohl Y, von Arx T. Decoronation of an ankylosed tooth for preservation of alveolar bone prior to implant placement. Dent Traumatol 2001 Apr;17(2):93-95.

12. Valdec S, Pasic P, Soltermann A, Thoma D, Stadlinger B, Rücker M. Alveolar ridge preservation with autologous particulated dentin—a case series. Int J Implant Dent 2017 Dec;3(1):12.

13. Schwarz F, Golubovic V, Becker K, Mihatovic I. Extracted tooth roots used for lateral alveolar ridge augmentation: a proof-of-concept study. J Clin Periodontol 2016 Apr;43(4): 345-353.

14. Kim YK, Lee J, Um IW, Kim KW, Murata M, Akazawa T, Mitsugi M. Tooth-derived bone graft material. J Korean Assoc Oral Maxillofac Surg 2013 Jun;39(3):103-111. 